\title{
Claves prácticas para un trasplante de microbiota fecal por colonoscopía en infección por Clostridium difficile recurrente. Experiencia en un centro universitario
}

\author{
Ricardo Cruz', Hugo Monrroy', Jorge Flandez', Carlos M. Pérez², \\ Manuel Álvarez-Lobos' y Cristian Hernández-Rocha'
}

\begin{abstract}
1 Departamento de Gastroenterología. Departamento de Enfermedades Infecciosas del Adulto. Pontificia Universidad Católica de Chile, Santiago, Chile. Escuela de Medicina.

Este trabajo fue financiado por el Fondo Nacional de Ciencia y Tecnología de Chile (FONDECYT) de inicio 11130502 de C.H.-R. y por la Vicerrectoría de Investigación de la Pontificia Universidad Católica de Chile (Proyecto Puente P1715/2017 de M.A.-L.).

Los autores declaran no tener conflicto de interés alguno.
\end{abstract}

Recibido: 20 de octubre de 2017 Aceptado: 2 de septiembre de 2018

Correspondencia a: Cristian Hernández-Rocha cristian.hernandez.rocha@gmail.

\section{Practical clues for a fecal microbiota transplantation by colonoscopy for recurrent Clostridium difficile infection. Experience in a University center}

Fecal microbiota transplantation (FMT) is a highly effective therapy in recurrent Clostridium difficile. The best route to administrate the fecal matter has not been established yet. However, the lower gastrointestinal route by colonoscopy is effective and safe, presenting a higher acceptance by patients. In addition, this route allows an evaluation of colonic mucosa seeking for differential diagnostics. We present a case series of FMT performed in our institution by colonoscopy, highlighting outcomes and practical aspects for its implementation.

Keywords: Clostridium difficile, microbiota, fecal microbiota transplantation, colonoscopy.

Palabras clave: Clostridium difficile, microbiota, trasplante de microbiota fecal, colonoscopia.

\section{Introducción}

$\checkmark$ 1 trasplante de microbiota fecal (TMF) consiste en la administración de material fecal desde una persona sana (donante), a un paciente con una enfermedad o condición relacionada con una alteración de la microbiota intestinal normal (disbiosis) ${ }^{1}$. La manipulación de la microbiota intestinal representa una prometedora estrategia para enfermedades digestivas y extra-digestivas donde la microbiota se encuentra alterada; sin embargo, la infección por Clostridium difficile (ICD) recurrente, caracterizada por una profunda disbiosis, constituye actualmente la principal indicación de $\mathrm{TMF}^{2,3}$. La alta efectividad del TMF, con respuesta superior a $80 \%$ en revisiones sistemáticas en el escenario de ICD recurren$\mathrm{te}^{4}$, ha estimulado el interés en esta terapia de pacientes, médicos, investigadores y la industria farmacéutica. De esta forma, actualmente el TMF ya no es considerado un recurso excepcional en la ICD recurrente y es cada vez más comúnmente practicado.

Existen diferentes rutas para la administración del material fecal: ruta alta a través de sonda naso-duodenal, naso-gástrica o gastroscopía; y ruta baja a través de colonoscopía total o enemas. Entre ellas, el TMF por colonoscopía ha demostrado ser altamente efectivo en la ICD recurrente $^{4}$ con una baja tasa de efectos adversos $(\mathrm{EA})^{5}$. Además, posee la ventaja potencial de evaluar la mucosa del colon en búsqueda de diagnósticos alternativos ante una diarrea persistente (enfermedad inflamatoria intestinal y otras causas infecciosas) y una mayor aceptación por parte de los pacientes ${ }^{6}$.

Si bien se han desarrollado otros métodos de entrega del TMF que resultan prometedores, los que usan cápsulas orales de deposiciones congeladas ${ }^{7-10}$, preparados orales de bacterias seleccionadas ${ }^{11-13}$ o la transferencia de filtrado fecal esteril ${ }^{14}$, éstos no se encuentran ampliamente disponibles. De esta forma, la creciente disponibilidad en nuestro medio de colonoscopía hace posible el acceso a TMF por esta vía como una alternativa útil al tratamiento de la ICD recurrente.

El objetivo de este artículo es mostrar nuestra experiencia en TMF por colonoscopía y destacar sus indicaciones y efectos adversos, selección y cribado del donante, así como aspectos prácticos en la realización del procedimiento.

\section{Indicaciones de trasplante de microbiota fecal}

Las indicaciones de TMF actualmente aceptadas $\operatorname{son}^{3,15-18}$ :

- ICD recurrente

a. Tres o más episodios de ICD leves a moderadas $\mathrm{y}$ falla de al menos un curso de tratamiento con vancomicina en dosis decreciente/pulso; con o sin el uso de un antimicrobiano alternativo (rifaximina o fidaxomicina).

b. Al menos dos episodios de ICD que resultaron en hospitalización y asociados a morbilidad significativa. 
Tabla 1. Contraindicaciones absolutas para ser donante de deposiciones

Riesgo de agentes infecciosos

Co-morbilidades gastrointestinales

Factores que alteran la composición de la microbiota

Otros
Infección conocida por VIH, hepatitis B o C

Exposición conocida a VIH y hepatitis viral dentro de los 12 meses previos

Conducta sexual de alto riesgo

Uso de drogas ilícitas

Tatuaje o piercing los seis meses previos

Historia de encarcelamiento

Enfermedad transmisible actual (ej.: infección respiratoria)

Factores de riesgo para Creutzfeldt-Jacob

Viaje a áreas endémicas para diarrea del viajero dentro de los últimos seis meses

Historia de enfermedad inflamatoria intestinal

Historia de síndrome de intestino irritable, constipación crónica o diarrea crónica

Historia de cáncer gastrointestinal o pólipos

Uso de antimicrobianos los tres meses previos

Uso de medicamentos inmunosupresores o agentes antineoplásicos

Reciente ingestión de alergenos a los cuales el receptor tiene alergia conocida

VIH, virus de la inmunodeficiencia humana.

- ICD refractaria.

c. ICD moderada sin respuesta a terapia estándar (vancomicina o fidaxomicina) por al menos una semana.

d. ICD grave sin respuesta a terapia estándar a las $48 \mathrm{~h}$.

El TMF en pacientes con ICD recurrente ha sido avalado por múltiples estudios clínicos randomizados y revisiones sistemáticas ${ }^{19-22}$. En cambio, en ICD grave y refractaria, la recomendación está basada sólo en reportes de casos y series retrospectivas, siendo más débil su indicación ${ }^{23-27}$.

\section{Descripción del procedimiento y claves prácticas para un trasplante de microbiota fecal por colonoscopía}

\section{El donante}

La elección del mejor donante aún es controversial. Las opciones abarcan desde heces congeladas de donantes anónimos recolectadas en un banco, hasta la donación de heces frescas de un familiar que vive bajo el mismo techo. La primera tiene las ventajas de la seguridad debido a un extenso cribado de enfermedades infecciosas y no infecciosas, procesamiento estandarizado y rápida disponibilidad. Sin embargo, son escasos los centros que actualmente disponen de estos bancos de deposiciones. Por otro lado, el donante familiar con contacto estrecho (p. ej.: cónyuge u otros familiares) tendría las ventajas potenciales de compartir factores de riesgo ambientales minimizando el riesgo de transmisión de enfermedades infecciosas. Sin embargo, hasta ahora no se ha evaluado si el uso de bancos de material fecal o el trasplante a partir de donantes relacionados representa un mayor nivel de seguridad para el receptor ${ }^{1}$.

Cuando no se cuenta con un banco de deposiciones y se elige un donante conocido, es importante hacer un cribado de enfermedades potencialmente transmisibles a través del material fecal.

El proceso de evaluación se inicia con una acabada historia clínica. Se recomienda el uso de cuestionarios similares a los utilizados en donantes de sangre. Las Tablas 1 y 2 muestran las contraindicaciones absolutas y relativas del candidato a donante y que deben ser evaluadas en la historia clínica ${ }^{15}$. Estas contraindicaciones no sólo incluyen enfermedades infecciosas, sino también enfermedades o condiciones que podrían teóricamente ser trasmitidas a través de la microbiota (p. ej.: enfermedad inflamatoria intestinal, síndrome de intestino irritable, trastornos metabólicos y autoinmunes). Los donantes deben evitar el consumo de alimentos a los que el receptor es alérgico al menos por cinco días previos al procedimiento.

Tabla 2. Contraindicaciones relativas para ser donante de deposiciones

Historia de cirugía gastrointestinal mayor (ej: cirugía bariátrica)

Síndrome metabólico (obesidad)

Enfermedad autoinmune (ej: esclerosis múltiple, lupus sistémico)

Enfermedades atópicas (asma, atopía, trastornos digestivos eosinofílicos)

Síndromes dolorosos crónicos (ej: fibromialgia, síndrome de fatiga crónica) 


\begin{tabular}{|c|c|c|c|}
\hline \multicolumn{4}{|c|}{ Exámenes mínimos } \\
\hline Deposiciones & Sangre & Considerar & Posiblemente \\
\hline \multirow{2}{*}{$\begin{array}{l}\text { - Clostridium difficile } \\
\text { (idealmente por RPC) }\end{array}$} & - ELISA para VIH 1 y 2 & - Giardia lamblia & - Citomegalovirus \\
\hline & - IgM para VHA & - Amebas & - HTLV-1 \\
\hline - Coprocultivo corriente & - HBsAg & - Criptosporidium y Ciclospora & - Virus de Epstein-Barr \\
\hline \multirow{9}{*}{$\begin{array}{l}\text { - Parasitológico en deposiciones } \\
\text { (idealmente tres muestras) }\end{array}$} & - Anticuerpos para VHC & - Escherichia coli 0157 & - Amebas \\
\hline & - RPR & - Rotavirus & - Isospora sp \\
\hline & & - Listeria monocytogenes & - Blastocystis hominis \\
\hline & & - Vibrio spp & - Strongiloides stercolaris \\
\hline & & - Norovirus & - Helicobacter pylori \\
\hline & & \multirow{4}{*}{$\begin{array}{l}\text { Considerar el uso de RPC multipatógenos } \\
\text { en deposiciones }\end{array}$} & - Schistosoma sp \\
\hline & & & - Virus JC \\
\hline & & & - Enterococo resistente a vancomicina \\
\hline & & & $\begin{array}{l}\text { - Staphylococcus aureus resistente a } \\
\text { meticilina }\end{array}$ \\
\hline
\end{tabular}

RPC: reacción de polimerasa en cadena; VIH: virus de la inmunodeficiencia humana; IgM: inmunoglobulina M; VHA: virus de la hepatitis A; HBsAg: antígeno de superficie de la hepatitis B, VHC: virus de la hepatitis C; RPR: Rapid plasma reagin; HTLV-1: virus linfotropo humano tipo 1; JC: John Cunningham. Tabla modificada de referencia 1.

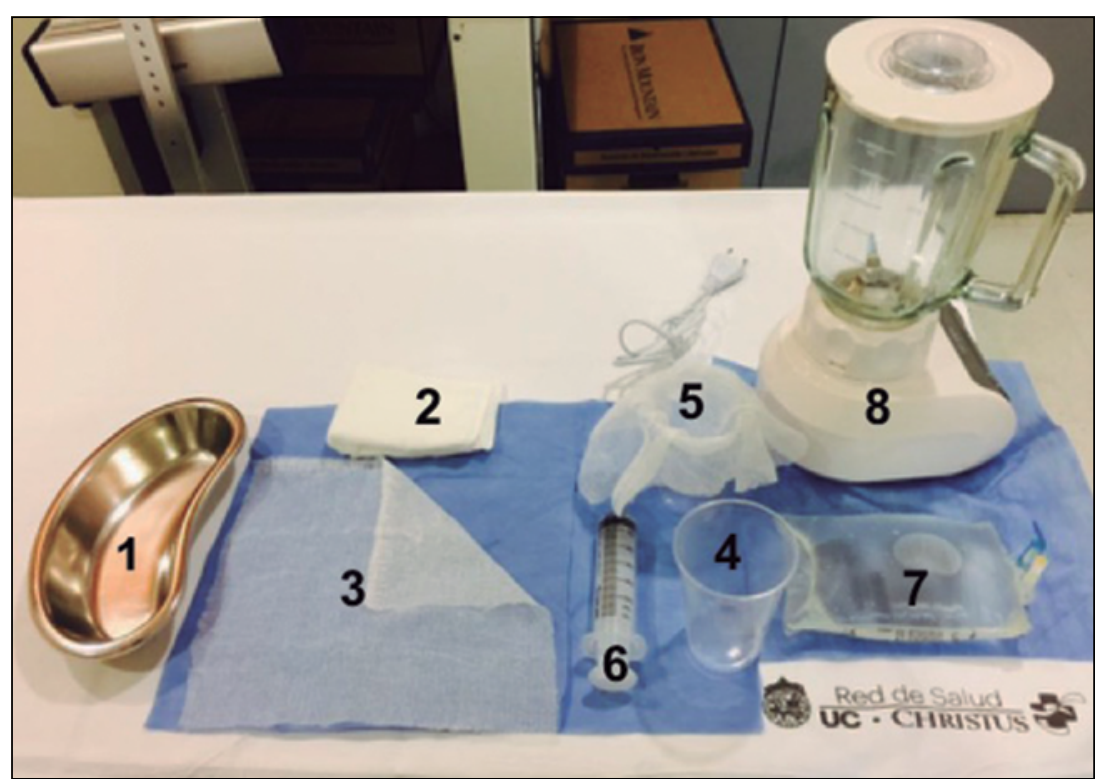

Figura 1. Implementos básicos para el trasplante de microbiota fecal por colonoscopía. 1, riñón para recoletar el material fecal; 2, apósito ultilizado para obtener la gasa porosa; 3, gasa porosa abierta; 4, vaso de 350 cc para recolectar el licuado de deposiciones; 5, vaso con gasa porosa en posición para filtrar las deposiciones licuadas; 6 ; jeringa punta roma de 50 cc para administrar el filtrado través del canal de trabajo del colonoscopio; 7, solución salina normal 500 cc; 8, juguera o licuadora.

Los exámenes de cribado del donante se muestran en la Tabla 3. Se detallan los exámenes mínimos para todo potencial donante. La solicitud de otros exámenes debe considerarse de acuerdo al riesgo del receptor (p. ej.: paciente inmunosuprimido) y al riesgo de exposición del donante evaluado en la historia clínica ${ }^{1}$. También es una consideración para la extensión del estudio, lo estrecho de la relación entre donante-receptor y la urgencia con la que se necesita el TMF. Un estudio reciente sugiere descartar sobre-crecimiento bacteriano intestinal en el donante; sin embargo, se requiere más evidencia para hacer esta evaluación mandatoria pre-trasplante ${ }^{28}$. Es responsabilidad del médico que realiza el TMF realizar la historia clínica, así como la solicitud y revisión de los exámenes de cribado.

El día del TMF es importante evaluar si el donante ha presentado en los días previos o presenta actualmente síntomas sugerentes de infección como fiebre, diarrea y/o vómitos, con el fin de evitar la transmisión de infecciones no detectadas durante el cribado.

\section{El paciente}

La entrega de información detallada sobre el procedimiento, sus beneficios y riesgos, la preparación del paciente, así como la obtención del consentimiento informado es un paso fundamental en el TMF. Algunos expertos recomiendan la realización de exámenes de cribado básico de enfermedades infecto-contagiosas al receptor para conocer su condición basal; sin embargo, esto es opcional $^{15}$. En el caso de ICD recurrente, el paciente se encuentra generalmente en tratamiento con vancomicina, la que debe suspenderse $48 \mathrm{~h}$ antes del TMF. 
El TMF por colonoscopía puede ser realizado en forma ambulatoria u hospitalizado dependiendo de la condición del paciente.

La preparación para la colonoscopía es la habitual, prefiriendo en nuestro grupo la administración de cuatro litros de polietilenglicol en dosis dividida. La dosis más cercana al procedimiento no debería ser administrada más de cinco horas antes de la colonoscopía para asegurar una mejor limpieza del colon ${ }^{29}$. Una inadecuada preparación dificulta el procedimiento y algunos autores plantean que podría disminuir la efectividad del transplante ${ }^{30}$.

\section{El procedimiento}

La preparación de las deposiciones del donante debería ser realizada inmediatamente antes de iniciar la colonoscopía. El preparado se debe utilizar dentro de las $24 \mathrm{~h}$ e idealmente dentro de las primeras seis horas ${ }^{15}$. En la Figura 1 se señalan algunos de los materiales necesarios para la preparación del TMF por colonoscopía. La preparación de las deposiciones debe considerar las medidas de protección para la manipulación de material biológico, incluyendo uso de guantes, pechera y visera de todo el personal que participa en el procedimiento.

En nuestro grupo solicitamos al receptor o donante que asista con una licuadora comercial nueva cuyo contenedor debe ser idealmente de plástico para facilitar su eliminación. El donante defeca en un riñón de cartón, metal o plástico estéril y luego este material es entregado en la unidad de endoscopía para su procesamiento en los plazos previamente descritos. La cantidad de material fecal recomendada es entre 20 y $100 \mathrm{~g}^{17}$. El uso por parte del donante de un laxante como lactulosa la noche previa al TMF no está evaluado en la literatura médica, pero podría ayudar a la obtención de una muestra más abundante en algunos casos. Se debe tener cuidado con potenciales efectos adversos como diarrea o distensión abdominal.

Las deposiciones recolectadas son licuadas y homogenizadas en la licuadora con 200 a 500 cc de solución salina fisiológica ( $\mathrm{NaCl} 9 \%$ ), agua de la llave o leche. No existen estudios que demuestren diferencias en efectividad con diferentes preparaciones; sin embargo, la solución salina fisiológica podría preservar mejor la microbiota ${ }^{1}$.

La muestra homogeneizada es filtrada de detritus sólidos con una gasa porosa (ej: gasa de los apósitos) o un filtro de café, a la vez que es depositada en vasos plásticos transparentes de 350 o 500 cc para su posterior infusión.

La colonoscopía es realizada con la técnica habitual. Idealmente la muestra licuada y homogeneizada debe ser infundida desde el íleon distal, el que ha sido identificado como reservorio de $C$. difficile ${ }^{31}$. La infusión se realiza con jeringas con "punta roma" de $50 \mathrm{~mL}$ a través del canal de trabajo del colonoscopio desde el íleon y ciego hasta el recto. Se recomienda realizar la instilación en sentido antigravitacional para asegurar la exposición de la mayor superficie de mucosa a la infusión. Se utilizan aproximadamente 50 a $150 \mathrm{~mL}$ en cada segmento del colon y al terminar la infusión, en cada segmento se debe aspirar el aire (evitando la aspiración del material instilado) para permitir el contacto de las superficies. En áreas con divertículos, la infusión debería ser particularmente generosa. En algunos pacientes, el uso de una dosis de 2 mg de loperamida oral inmediatamente antes del procedimiento podría ayudar a la mejor retención del material trasplantado; sin embargo, su uso no ha sido evaluado en estudios clínicos y es opcional ${ }^{15}$.

\section{Efectos adversos}

Los potenciales efectos adversos (EA) del TMF pueden ser clasificados como de corto y largo plazo. Los primeros a su vez pueden ser clasificados como aquellos relacionados al método de entrega del TMF o aquellos relacionados al TMF propiamente tal ${ }^{1}$. Los EA menores a corto plazo son comunes e incluyen dolor abdominal, distensión, flatulencia, diarrea o fiebre transitoria. Los EA graves, muy poco frecuentes, son los secundarios al procedimiento usado para realizar el TMF e incluyen: perforación, hemorragia, depresión cardio-respiratoria por la sedación utilizada y casos anecdóticos de neumonía aspirativa con mortalidad asociada ${ }^{5}$. También se ha descrito la transmisión de agentes infecciosos y bacteriemia ${ }^{5,32}$. Los EA a largo plazo tienen relación con el potencial de la microbiota intestinal de generar o modular ciertas enfermedades. Algunas condiciones que han sido ligadas a una alteración de la microbiota intestinal incluyen: obesidad, diabetes mellitus, ateroesclerosis, hígado graso, enfermedad inflamatoria intestinal, síndrome de intestino irritable, asma y autismo. Estudios a largo plazo definirán mejor el perfil de riesgo a largo plazo del $\mathrm{TMF}^{1}$.

\section{Descripción de nuestra serie}

Entre noviembre de 2013 y diciembre de 2016 se han realizado ocho TMF por colonoscopía en el Hospital Clínico de la Pontificia Universidad Católica de Chile.

Todos los TMF fueron realizados por ICD recurrente. Las características de los pacientes se describen con mayor detalle en la Tabla 4. La media de edad fue 47,4 años (rango, 23-72 años), siendo siete mujeres (87,5\%). En todos los casos se realizó el TMF por colonoscopía con preparación previa con polietilenglicol, cuatro litros en dosis dividida. Las colonoscopías fueron ambulatorias en seis pacientes. Los dos pacientes en que el procedimiento se hizo hospitalizado fue debido a sus co-morbilidades.

La sedación utilizada fue midazolam y fentanilo en dosis estándar en seis procedimientos. Dos procedimientos fueron realizados con propofol y asistencia de anestesista por preferencia de los pacientes. No se encontraron hallaz- 


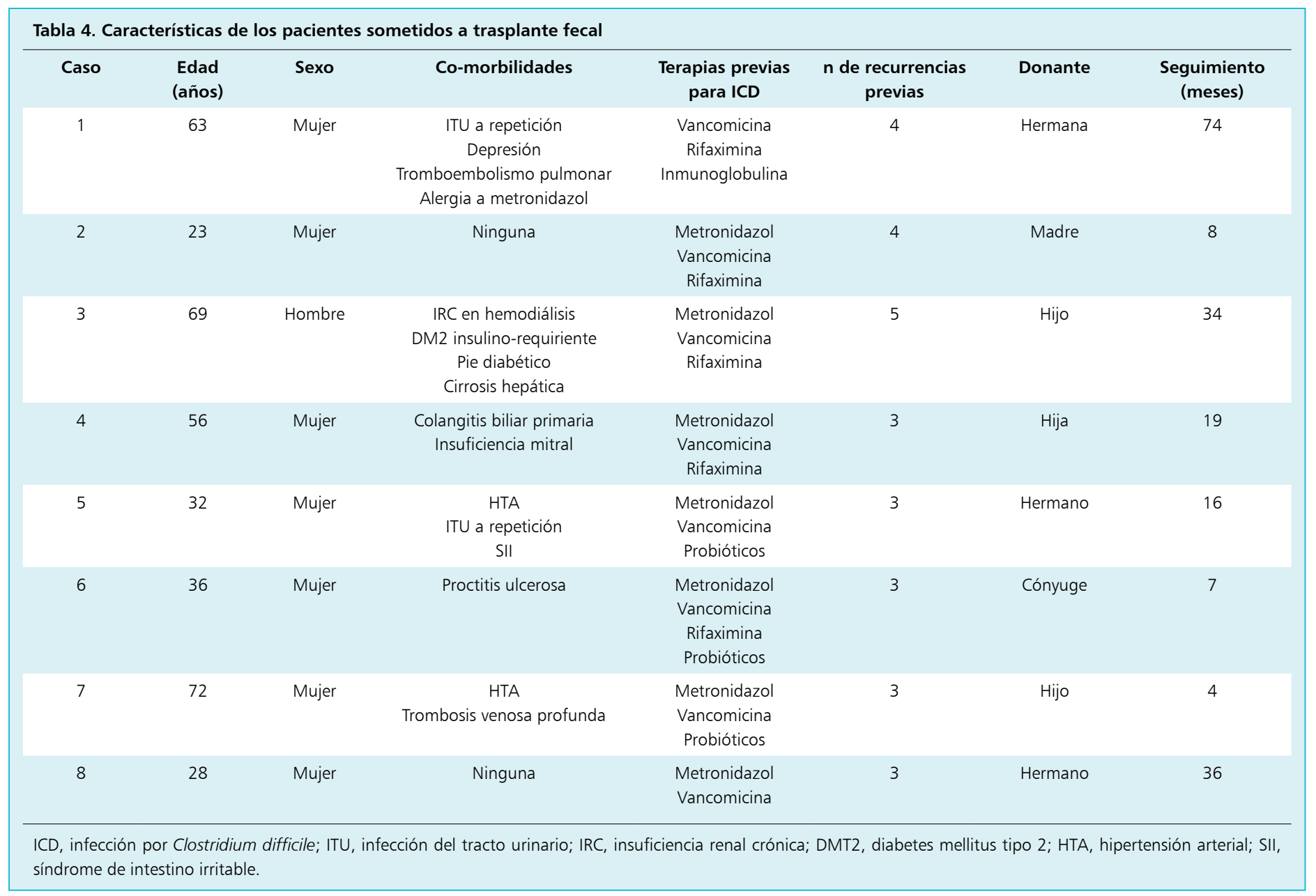

gos relevantes en las colonoscopías realizadas, excepto por una proctitis leve en uno de los pacientes con colitis ulcerosa concomitante.

El cribado de los donantes, quienes eran familiares de primer grado en siete casos y el cónyuge en un caso, incluyó: reacción de polimerasa en cadena (RPC) para $C$. difficile, coprocultivo corriente y parasitológico en deposiciones (tres muestras). En sangre se solicitó serología (ELISA) para virus de la inmunodeficencia humana (VIH) 1 y 2, IgM para virus hepatitis A (VHA), antígeno de superficie para virus hepatitis B ( $\mathrm{HBsAg})$, serología (ELISA) para virus hepatitis C (VHC) y RPR (rapid plasma reagin).

Ninguno de los donantes utilizó lactulosa y en dos receptores se usó loperamida $2 \mathrm{mg}$ vía oral inmediatamente después del procedimiento dada la imposibilidad de retener el material fecal trasplantado. La preparación de las heces en todos los pacientes se realizó con solución salina fisiológica a través de la técnica descrita previamente.
No se registraron EA graves durante o posteriores al procedimiento. Dos pacientes presentaron dolor abdominal tipo cólico con posteriorioridad al procedimiento, lo que fue manejado con antiespasmódicos endovenosos con buena respuesta.

La mediana de seguimiento de los pacientes fue de 17,5 meses (rango, 4-74 meses). Uno de los pacientes presentó dos años después del TMF un cuadro de diarrea acuosa de una semana de evolución tras usar ciprofloxacina a raíz de una infección en pie diabético. La RPC para $C$. difficile fue positiva; sin embargo, al momento del control el paciente se encontraba asintomático por lo que no fue tratado. Doce meses luego de ese episodio el paciente no ha presentado nuevos cuadros de ICD a pesar del uso reiterado de antimicrobianos por similar causa. En dos pacientes ha sido necesario el uso de antimicrobianos por otros motivos, sin registrarse nuevos episodios de ICD. Dos pacientes evolucionaron con síntomas compatibles con síndrome de intestino irritable (SII) post-infeccioso 
(uno de ellos con antecedente de síntomas digestivos funcionales previos al TMF) y han sido manejados con tratamiento sintomático.

\section{Discusión}

La microbiota intestinal está involucrada en la patogenia de múltiples enfermedades intestinales y extraintestinales. De esta forma, el trasplante de microbiota fecal ha sido visto como alternativa de tratamiento en enfermedades como: encefalopatía hepática, trastornos del espectro autista, síndrome de intestino irritable, enfermedad inflamatoria intestinal, trastornos metabólicos, etc. Sin embargo, la evidencia más sólida de su beneficio ha sido observada en el tratamiento de la ICD recurrente ${ }^{2}$.

La ICD recurrente constituye una patología desafiante en su manejo y con altos costos para los pacientes e instituciones de salud. El TMF es un tratamiento altamente efectivo y seguro en la ICD recurrente, tanto por ruta alta como baja por colonoscopía mostrando una efectividad de $87 \%$ siguiendo el primer $\mathrm{TMF}^{4}$. Sin embargo, evidencia más reciente muestra que hasta $30 \%$ de los pacientes podrían requerir un segundo TMF para lograr la resolución de la infección, siendo factores predictores de falla del primer transplante los casos graves de ICD y la mala preparación de la colonoscopía ${ }^{30}$.

Asimismo, aún existe discusión sobre la ruta más efectiva y segura para aplicar esta terapia ${ }^{4,5,19,33}$. A la fecha hay dos estudios de diseño controlado randomizado que evalúan la efectividad del TMF en ICD recurrente. El primero, que usó la administración a través de sonda naso-duodenal de material fecal demostró una tasa de cura de $81 \%$ del TMF comparado con $31 \%$ de un tratamiento estándar con vancomicina oral ${ }^{34}$. El segundo estudio, recientemente publicado, comparó la administración a través de colonoscopía de TMF heterólogo (de donante) o autólogo (deposiciones del mismo enfermo), siendo la efectividad de 90,2 y $62,5 \%$, respectivamente ${ }^{35}$. En una revisión sistemática que analizó 20 estudios con un total de 536 pacientes y que incluyó el primer estudio randomizado mencionado, se observó que la eficacia del TMF realizado por ruta baja fue discretamente superior (84-93\%) a la ruta alta (81-86\%) en ICD recurrente ${ }^{4}$. Esta diferencia no fue observada en un pequeño estudio piloto que comparó ambas modalidades ${ }^{36}$.

Nuestros datos destacan también esta alta efectividad observada con el TMF realizado por colonoscopía en ICD recurrente, destacando que todos los pacientes fallaron a múltiples terapias con antimicrobianos, incluyendo vancomicina en dosis en desescalamiento. Antimicrobianos como metronidazol y vancomicina si bien son efectivos contra $C$. difficile, al igual que otros antimicrobianos, alteran la microbiota intestinal perpetuando la disbiosis y consecuentemente el riesgo de ICD recurrente ${ }^{37}$. Alternativas como el antimicrobiano fidaxomicina han demostrado disminuir el riesgo de disbiosis y recurrencia ${ }^{38}$; sin embargo, su costo y disponibilidad han dificultado un uso más amplio.

Con respecto a las complicaciones, no existe aún claridad de la ruta más segura para el TMF. Los EA globales atribuibles al TMF fueron más frecuentes en la ruta alta $(43,6 \%)$ comparada con la baja $(17,7 \%)$ en una revisión sistemática. Sin embargo, la ruta baja presentó mayor frecuencia de EA graves (6,1 versus 2\%). Los autores de esta revisión destacan que un porcentaje menor de los EA graves, estuvieron relacionados definitivamente al $\mathrm{TMF}^{5}$; además, otras revisiones sistemáticas han encontrado más bajas tasas de complicaciones, sin diferencias significativas en EA según la ruta de administración ${ }^{18,32}$.

Recientemente, un estudio retrospectivo evaluó la seguridad del TMF en pacientes inmunosuprimidos, demostrando ser una terapia segura en este grupo de pacientes $^{39}$. Nuestra serie de pacientes va en línea con estos datos, reforzando la seguridad del procedimiento y su aplicabilidad en nuestro medio. Sin embargo, seguimientos a largo plazo de un gran número de pacientes sometidos a TMF son necesarios para evaluar la aparición de EA menos frecuentes, sobre todo de la esfera autoinmune y metabólica.

Cabe destacar que en todos nuestros pacientes la indicación fue ICD recurrente y el TMF fue realizado en forma electiva, no incluyendo pacientes con ICD grave refractaria. La indicación y seguridad en este último escenario no ha sido claramente establecida. En nuestra serie, dos pacientes desarrollaron SII post-infeccioso el cual podría ser secundario a la ICD más que al TMF. Esto se basa en recientes estudios que muestran una alta frecuencia de SII posterior a la ICD, sobre todo cuando la infección es de larga duración ${ }^{40}$.

En esta revisión destacamos los aspectos prácticos del TMF por colonoscopía en cada una de sus fases. Si este procedimiento se realiza teniendo las consideraciones mencionadas, resulta ser sencillo y los materiales para su realización se encuentran disponibles en cualquier unidad de endoscopía. Se ha argumentado que el TMF por endoscopía alta o baja podría aumentar el uso de recursos en salud; sin embargo, un estudio de costo-efectividad determinó que el TMF por endoscopía es menos costoso y más efectivo que el tratamiento con vancomicina, incluso como terapia del primer episodio de la $\mathrm{ICD}^{41}$. Sin embargo, no se puede olvidar que el mayor desafío en la aplicación del TMF está en la correcta indicación del procedimiento, conociendo su efectividad y riesgos (tanto del TMF como la colonoscopía), la meticulosa elección y cribado del donante y la experiencia en la técnica de endoscopía digestiva baja. Teniendo en cuenta estas 
consideraciones y reconociendo los aspectos prácticos para su realización, el TMF podría ser realizado en forma limpia y segura para el paciente.

\section{Resumen}

El trasplante de microbiota fecal (TMF) constituye una terapia altamente eficaz en la infección por Clostridium difficile (ICD) recurrente. La mejor vía de administración del material fecal aún no ha sido establecida; sin embargo, la vía baja a través de colonoscopía resulta eficaz, segura y de mayor aceptación por los pacientes, permitiendo además el examen de la mucosa del colon en busca de diagnósticos diferenciales. Presentamos una serie de casos de TMF realizados en nuestra institución a través de colonoscopía, destacando los resultados y aspectos prácticos para su implementación.

\section{Referencias bibliográficas}

1.- Kelly C R, Kahn S, Kashyap P, Laine L, Rubin D, Atreja A, et al. Update on fecal microbiota transplantation 2015: indications, methodologies, mechanisms, and outlook. Gastroenterology 2015; 149: 223-37. doi: 10.1053/j.gastro.2015.05.008.

2.- Sha S, Liang J, Chen M, Xu B, Liang C, Wei $\mathrm{N}$, et al. Systematic review: faecal microbiota transplantation therapy for digestive and nondigestive disorders in adults and children. Aliment Pharmacol Ther 2014; 39: 1003-32. doi: 10.1111/apt.12699.

3.- McDonald L C, Gerding D N, Johnson S, Bakken J S, Carroll K C, Coffin S E, et al. Clinical practice guidelines for Clostridium difficile infection in adults and children: 2017 Update by the Infectious Diseases Society of America (IDSA) and Society for Healthcare Epidemiology of America (SHEA).Clin Infect Dis 2018; 66: 987-94. doi: 10.1093/cid/ cix 1085 .

4.- Cammarota G, Ianiro G, Gasbarrini A. Fecal microbiota transplantation for the treatment of Clostridium difficile infection: a systematic review. J Clin Gastroenterol 2014; 48: 693-702. doi: 10.1097/MCG.0000000000000046.

5.- Wang S, Xu M, Wang W, Cao X, Piao M, Khan $\mathrm{S}$, et al. Systematic review: adverse events of fecal microbiota transplantation. PLoS ONE 2016; 11: e0161174. doi: 10.1371/journal. pone. 0161174 .

6.- Zipursky J S, Sidorsky T I, Freedman C A, Sidorsky M N, Kirkland K B. Patient attitudes toward the use of fecal microbiota transplantation in the treatment of recurrent Clostridium difficile infection. Clin Infect Dis 2012; 55: 1652-8. doi: 10.1093/cid/cis809.

7.- Youngster I, Russell G H, Pindar C, Ziv-Baran T, Sauk J, Hohmann EL. Oral, capsulized, frozen fecal microbiota transplantation for relapsing Clostridium difficile infection. JAMA 2014; 312: 1772-8. doi: 10.1001/ jama.2014.13875.

8.- Youngster I, Mahabamunuge J, Systrom H K, Sauk J, Khalili H, Levin J, et al. Oral, frozen fecal microbiota transplant (FMT) capsules for recurrent Clostridium difficile infection. BMC Med 2016; 14: 134. doi: 10.1186/s12916-0160680-9.

9.- Hecker M T, Obrenovich M E, Cadnum J L, Jencson A L, Jain A K, Ho E, et al. Fecal microbiota transplantation by freeze-dried oral capsules for recurrent Clostridium difficile infection. Open Forum Infect Dis 2016; 3: ofw091. DOI:10.1093/ofid/ofw091.

10.- Tang G, Yin W, Liu W. Is frozen fecal microbiota transplantation as effective as fresh fecal microbiota transplantation in patients with recurrent or refractory Clostridium difficile infection: A meta-analysis? Diagn Microbiol Infect Dis 2017; 88: 322-9. doi: 10.1016/j. diagmicrobio.2017.05.007

11.- Tvede M, Rask-Madsen J. Bacteriotherapy for chronic relapsing Clostridium difficile diarrhoea in six patients. The Lancet 1989; 1: 1156-60.

12.- Petrof E O, Gloor G B, Vanner S J, Weese $\mathrm{S} \mathrm{J}$, Carter D, Daigneault M C, et al. Stool substitute transplant therapy for the eradication of Clostridium difficile infection: "RePOOPulating" the gut. Microbiome 2013; 1: 3. doi: 10.1186/2049-2618-1-3.

13.- Khanna S, Pardi D S, Kelly C R, Kraft C S, Dhere T, Henn M R, et al. A novel microbiome therapeutic increases gut microbial diversity and prevents recurrent Clostridium difficile infection. J Infect Dis 2016; 214: 173-81. doi: 10.1093/infdis/jiv766.

14.- Ott S J, Waetzig G H, Rehman A, MoltzauAnderson J, Bharti R, Grasis J A, et al. Efficacy of sterile fecal filtrate transfer for treating patients with Clostridium difficile infection. Gastroenterology 2017; 152: 799-811.e7. doi: 10.1053/j.gastro.2016.11.010.

15.- Bakken J S, Borody T, Brandt L J, Brill J V, Demarco D C, Franzos M A,et al. Treating Clostridium difficile infection with fecal microbiota transplantation. Clin Gastroenterol Hepatol 2011; 9: 1044-9. doi: 10.1016/j. cgh.2011.08.014

16.- Surawicz C M, Brandt L J, Binion D G, Ananthakrishnan A N, Curry S R, Gilligan P H, et al. Guidelines for diagnosis, treatment, and prevention of Clostridium difficile infections. Am J Gastroenterol 2013; 108: 478-98; quiz
499. doi: 10.1038/ajg.2013.4.

17.- Cammarota G, Ianiro G, Tilg H, RajilićStojanović M, Kump P, Satokari R, et al. European consensus conference on faecal microbiota transplantation in clinical practice. Gut 2017; 66: 569-80. doi: 10.1136/ gutjnl-2016-313017.

18 Hernández-Rocha C, Pidal P, Ajenjo MC, Quera R, Quintanilla M, Lubascher J, et al. Chilean consensus of prevention, diagnosis and treatment of Clostridium difficileassociated diarrhea. Rev Chilena Infectol 2016; 33: 98-118. doi: 10.4067/S071610182016000100020.

19.- Li Y T, Cai H F, Wang Z H, Xu J, Fang J Y. Systematic review with meta-analysis: long-term outcomes of faecal microbiota transplantation for Clostridium difficile infection. Aliment Pharmacol Ther 2016; 43: 445-57. doi: 10.1111/apt.13492.

20.- Rossen N G, MacDonald J K, de Vries E M, Haens G R D', de Vos W M, Zoetendal E G, et al. Fecal microbiota transplantation as novel therapy in gastroenterology: A systematic review. World J Gastroenterol 2015; 21: 535971. doi: 10.3748/wjg.v21.i17.5359.

21.- Dodin M, Katz D E. Faecal microbiota transplantation for Clostridium difficile infection. Int J Clin Pract 2014; 68: 363-8. doi: 10.1111/ijcp.12320.

22.- Kassam Z, Lee C H, Yuan Y, Hunt R H. Fecal microbiota transplantation for Clostridium difficile infection: systematic review and metaanalysis. Am J Gastroenterol 2013; 108: 500-8. doi: 10.1038/ajg.2013.59.

23.- You D M. Successful Treatment of fulminant Clostridium difficile infection with fecal bacteriotherapy. Ann Intern Med 2008; 148: 632.

24.- Weingarden A R, Hamilton M J, Sadowsky M J, Khoruts A. Resolution of severe Clostridium difficile infection following sequential fecal microbiota transplantation. J Clin Gastroenterol 2013; 47: 735-7. doi: 10.1097/ MCG.0b013e31829004ae.

25.- Fischer M, Sipe B W, Rogers N A, Cook G K, Robb B W, Vuppalanchi R, et al. Faecal microbiota transplantation plus selected 
use of vancomycin for severe-complicated Clostridium difficile infection: description of a protocol with high success rate. Aliment Pharmacol Ther 2015; 42: 470-6. doi: 10.1111/ apt. 13290 .

26.- Zainah H, Hassan M, Shiekh-Sroujieh L, Hassan S, Alangaden G, Ramesh M. Intestinal microbiota transplantation, a simple and effective treatment for severe and refractory Clostridium difficile infection. Dig Dis Sci 2015; 60: 181-5. doi: 10.1007/s10620-0143296-y.

27.- Fischer M, Sipe B, Cheng Y-W, Phelps E, Rogers N, Sagi S, et al. Fecal microbiota transplant in severe and severe-complicated Clostridium difficile: A promising treatment approach. Gut Microbes 2017; 8: 289-302. doi: 10.1080/19490976.2016.1273998.

28.- Allegretti J R, Kassam Z, Chan W W. Small intestinal bacterial overgrowth: should screening be included in the pre-fecal microbiota transplantation evaluation?. Dig Dis Sci 2018; 63: 193-7. doi: 10.1007/s10620-0174864-8.

29.- Parra-Blanco A, Ruiz A, Álvarez-Lobos M, Amorós A, Gana J C, Ibáñez P, et al. Achieving the best bowel preparation for colonoscopy. World J Gastroenterol 2014; 20: 17709-26. doi: 10.3748/wjg.v20.i47.17709.

30.- Ianiro G, Valerio L, Masucci L, Pecere S, Bibbò S, Quaranta G, et al. Predictors of failure after single faecal microbiota transplantation in patients with recurrent Clostridium difficile infection: results from a 3-year, single-centre cohort study. Clin Microbiol Infect 2017; 23: 337.e1-337.e3. https://doi.org/10.1016/j. cmi.2016.12.025.

31.- Testore G P, Nardi F, Babudieri S, Giuliano M, Di Rosa R, Panichi G. Isolation of Clostridium difficile from human jejunum: identification of a reservoir for disease? J Clin Pathol 1986; 39: 861-2.

32.- Espinoza R, Quera R, Meyer L, Rivera D. Fecal microbiota transplantation: first case report in Chile and review. Rev Chilena Infectol 2014; 31: 477-82. doi: 10.4067/S071610182014000400016.

33.- Baxter M, Colville A. Adverse events in faecal microbiota transplant: a review of the literature. J Hosp Infect 2016; 92: 117-27. doi: 10.1016/j. jhin.2015.10.024.

34.- van Nood E, Vrieze A, Nieuwdorp M, Fuentes S, Zoetendal E G, de Vos W M, et al. Duodenal infusion of donor feces for recurrent Clostridium difficile. N Engl J Med 2013; 368: 407-15. doi: 10.1056/NEJMoa1205037.

35.- Kelly C R, Khoruts A, Staley C, Sadowsky M J, Abd M, Alani M, et al. Effect of fecal microbiota transplantation on recurrence in multiply recurrent Clostridium difficile infection: A randomized trial. Ann Intern Med 2016; 165: 609-16. doi: 10.7326/M16-0271.

36.- Youngster I, Sauk J, Pindar C, Wilson R G, Kaplan J L, Smith M B, et al. Fecal microbiota transplant for relapsing Clostridium difficile infection using a frozen inoculum from unrelated donors: a randomized, open-label, controlled pilot study. Clin Infect Dis 2014; 58: 1515-22. doi: 10.1093/cid/ciu135.

37 Louie T J, Byrne B, Emery J, Ward L, Krulicki W, Nguyen D, et al. Differences of the fecal microflora with Clostridium difficile therapies. Clin Infect Dis 2015; 60 Suppl 2: S91-7. doi: $10.1093 /$ cid/civ252.

38.- Cornely O A, Nathwani D, Ivanescu C, Odufowora-Sita O, Retsa P, Odeyemi I A O. Clinical efficacy of fidaxomicin compared with vancomycin and metronidazole in Clostridium difficile infections: a meta-analysis and indirect treatment comparison. J Antimicrob Chemother 2014; 69: 2892-900. doi: 10.1093/jac/dku261.

39.- Kelly C, Ihunnah C, Fischer M, Er K, Surawicz C, Afzali A, et al. Fecal microbiota transplant for treatment of Clostridium difficile infection in immunocompromised patients. Am J Gastroenterol. 2014 Jul;109(7): 1065-71. doi: 10.1038/ajg.2014.13.

40.- Wadhwa A, Nahhas M F Al, Dierkhising RA, Patel R, Kashyap P, Pardi D S, et al. High risk of post-infectious irritable bowel syndrome in patients with Clostridium difficile infection. Aliment Pharmacol Ther 2016; 44: 576-82. doi: 10.1111/apt.13737.

41.- Varier R U, Biltaji E, Smith K J, Roberts M S, Jensen M K, LaFleur J, et al. Cost-effectiveness analysis of treatment strategies for initial Clostridium difficile infection. Clin Microbiol Infect 2014; 20: 1343-51. doi: 10.1111/14690691.12805 . 